\title{
Estimation of Hydrogen Bond Donating Ability and the Dipolarity-Polarizability of Associated Water Molecules at the SDS Micellar Interface Using a Fluorescence Indicator
}

\author{
MRINMOY SHANNIGRAHI
}

Department of Physical Sciences, Sri Ramkrishna Sarada Siksha Mandir, Kamarpukur, Hooghly- 722161, India

mrinmoy.physchem@gmail.com

Received 21 December 2012 / Accepted 13 January 2013

\begin{abstract}
A new methodology for estimation of solvatochromic properties of micelles has been proposed. Two environment sensitive emission properties of a single indicator molecule have been used for this purpose. The hydrogen bond donating ability $(\alpha)$ and dipolarity- polarizability $\left(\pi^{*}\right)$ of associated water molecules at the SDS micellar interface has been estimated. The obtained result has been compared to the literature values. Effect of methodology and choice of indicator molecule has also been discussed.
\end{abstract}

Keywords: Micelles, Interfacial water, Solvatochromic properties, Fluorescence indicator

\section{Introduction}

Aqueous micellar media have widely been used in solubilising drug molecules ${ }^{1-2}$, catalysts for chemical reactions $s^{1-2}$, altering the selectivity of chromatographic ${ }^{3}$ and electrophoretic separation processes ${ }^{4}$. The interfacial region of the micelles has been used as a possible reaction site that can both accelerate and inhibit reactions compared to that in pure water ${ }^{5,6}$. Choice of proper reaction medium and understanding the selectivity of separation in micellar electrokinetic chromatography rests on the general solvating ability of the medium used. Thus the knowledge of solvatochromic properties in terms of Kamlet-Taft descriptors (namely $\pi^{*}, \alpha$ and $\beta$ ) for that medium is a prerequisite ${ }^{7}$. Recently some group of authors including the present author have successfully tried to estimate the solvatochromic properties of micellar media, namely H-bond donating $(\alpha)$ and acceptance abilities $(\beta)$, and the general solvating ability due to the dielectric effect i.e. dipolarity-polarisability $\left(\pi^{*}\right)^{8-13}$. A common practice for estimation of these properties for micellar media is the solvatochromic comparison method using Linear Solvation Energy Relationships (LSER) employing spectroscopic probe technique. For that purposes either specific indicator molecule has been used for estimation of a particular property ${ }^{8-10}$, or a series of structurally similar indicator molecules have been used ${ }^{11-13}$. However, the above procedures may be criticized for the following reasons: 
(i) Employing different types of indicator molecules for different solvatochromic properties rest on the assumption that they all probe the same region of micelle which may not be the case always in practice.

(ii) Use of several indicators for a particular property and then averaging over the estimated values has not been recommended recently for meaningful differences in chemistry of the indicators ${ }^{14}$. To avert this problem use of a single indicator molecule is proposed in the present work.

Two different but extremely environment sensitive emission properties (specifically the energy of fluorescence maximum, $E(F)$ and the non-radiative decay constant of the excited state, $k_{n r}$ ) of an indicator molecule ( a ketocyanine dye, Figure 1) has been used. Apart from high environment sensitivity, both the parameters have been found to be intensity independent i.e. no dependency on the dye concentration. Empirical correlations of these properties with the solvation properties of homogeneous media through LSER allow us to estimate the H-bond donating ability $(\alpha)$ and the dipolarity-polarizability $\left(\pi^{*}\right)$ of sodium dodecyl sulphate micellar phase. Although there are lot of other molecular probes like ET(30), prodan, coumarin 343 etc. that can sense two or more solvation parameters at the same time but no comprehensive effort has been made for simultaneous estimation of more than one property of micellar media using only one indicator molecule. Moreover the present methodology will help us to check the dependency of the solvatochromic properties on the choice of indicator molecule.

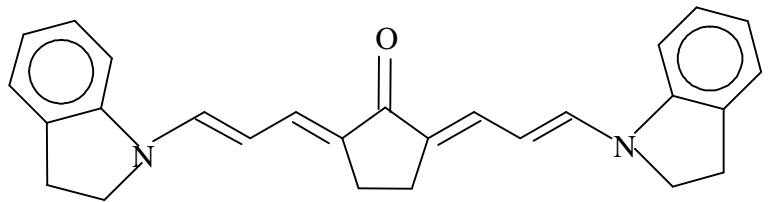

\section{Experimental}

Figure 1. Solvatochromic indicator molecule

The ketocyanine dye used in the present work has been prepared by the method described by Kessler and Wolfbeis ${ }^{15}$. All the solvents were purified by standard procedures and finally distilled over calcium hydride immediately prior to experiment to ensure the absence of moisture, peroxides and other oxidizing agents as impurities. Sodium dodecyl sulphate, SDS, was purchased from SIGMA and purified by repeated recrystallisation from ethanol. Triply distilled water was used for preparation of surfactant solutions.

\section{Spectroscopic measurements}

Steady-state fluorescence emission spectra were recorded on a HITACHI F-4500 spectrofluorimeter equipped with temperature controlled cell holder. In all the spectral measurements the concentration of the dye varied in the range $10^{-4}$ (surfactant solutions) to $10^{-5}$ (pure and mixed solvents) mol dm${ }^{-3}$. The excitation / emission slit widths were set to $2.5 \mathrm{~nm} / 2.5 \mathrm{~nm}$. The observed wavelength of maximum fluorescence has been converted to the energy unit by the simple relation:

$$
E(F)\left(\mathrm{kcal} \mathrm{mol}^{-1}\right)=28590 / \lambda(\mathrm{nm})
$$

The inaccuracy related to the measurement of $E(F)$ was found to be $\pm 0.1 \mathrm{kcal} / \mathrm{mol}$. Quantum yields corrected for refractive index were based on the ratio of the areas under the emission curves to that of Rhodamine B in ethanol; the quantum yield of the latter was taken as 0.71 . 
For excited state lifetime measurement, fluorescence decay was studied by Time Correlated Single Photon Counting (TCSPC) using the FLUOROCUBE life-time system (IBH, s/n 04412). A nano LED of $540 \mathrm{~nm}$ (IBH, UK, nano LED s/n 04225) was used as excitation source with FWHM of the order of $1 \mathrm{~ns}$ and the pulse is passed through $5000 \mathrm{M}$ emission monochromator. The fluorescence signal was detected in magic angle polarization $\left(54.70^{\circ}\right)$ using cooled red sensitive photocathode (TBX 04-A) detector with a response time of 180 ps. The decays were analyzed using IBH DAS-6 decay analysis software.

\section{Results and Discussion}

\section{Characteristics of the indicator molecule in homogeneous media}

Before the estimation of solvatochromic properties for micellar interface, it is advisable to study the spectroscopic properties of the indicator in homogeneous solvent media as solvatochromic comparison method has been adopted in the present work. Polyenic bis- $\omega, \omega$ amino ketones popularly known as ketocyanine dyes have been classified in the bichromophoric cyanine category with two $\omega$-aminopolyenic units bound to the central carbonyl oxygen. The electronic structure of the ketocyanine dye has been found to be a resonance between neutral and the charge-separated forms ${ }^{15}$. The drift of $\pi$-electrons (upon excitation) from the donor $R_{2} N$ to the acceptor $C=O$ is responsible for the charge separated form. This electronic transfer process can be controlled by suitable solvent. A polar protic environment will favour the charge separated form whereas solvent with lower polarity or no hydrogen bonding ability will favour the neutral form ${ }^{15-18}$. Solvatochromism thus exhibited by these dyes make them good probes for monitoring polarity and hydrogenbonding interactions. The solvation process of the indicator molecule in polar protic solvents like alcohols can be depicted in two steps:-

(i) Charge transfer upon electronic excitation to form the charge-separated $S_{1}$ state.

Resonance stabilization of the excited state makes the carbonyl oxygen more basic than its ground state.

(ii) In the next step, the highly basic carbonyl oxygen binds with the alcohols through $H$-bond formation.

The spectroscopic details of the indicator molecule have already been studied elaborately in pure solvents ${ }^{18}$, binary $^{19-21}$ and ternary ${ }^{22}$ solvent mixtures, micellar media ${ }^{21-24}$ and in cyclodextrin ${ }^{25}$ environment. The energy of maximum fluorescence, $E(F)$ for the indicator has been found to be very much sensitive towards the solvatochromic properties of medium. For a protic solvent, the maximum energy of transition is largely determined by the hydrogen-bond donating interactions between the hydrogen atom of the solvent and the carbonyl oxygen of the dye molecule. The greater the hydrogen-bonding ability of the medium, the lower is the energy of transition. The relationship between energy of maximum fluorescence and the solvent parameters can be empirically accounted by Multiple Linear Regression Analysis (MLRA) for 22 solvents (those having $\alpha \neq 0$ ) and mixed solvents (Table 1) by the following LSER equation:

$$
E(F)=(53.46 \pm 0.50)-(7.48 \pm 0.39) \alpha-(1.43 \pm 0.63) \pi^{*}
$$

From the above relation it is clearly evident that $\alpha$, the hydrogen-bond donating ability of the solvents plays a major contribution towards the energy of fluorescence of the dye molecule. We have already stated that resonance stabilized carbonyl oxygen of the dye molecule in the excited state forms tighter hydrogen-bonding with the protic solvents. To investigate the effect of medium on the dynamics of the excited state, the radiative $\left(k_{r}\right)$ and non-radiative $\left(k_{n r}\right)$ decay constants were calculated in a previous communication ${ }^{19}$ using the following expressions. 


$$
k_{r}=\varphi_{F} / \tau \text { and } k_{n r}=\tau^{-1}-k_{r}
$$

The study revealed that the radiative decay constant of the dye is practically insensitive towards the medium $\left(k_{r}=3.9 \times 10^{8}\right)$. However, non-radiative decay constant shows large environment sensitivity (Table 1). Once again the sensitivity can be expressed by the following LSER equation:

$$
\log k_{n r}=(10.11 \pm 0.15)-(0.87 \pm 0.12) \alpha-(0.78 \pm 0.19) \pi^{*}
$$

\begin{tabular}{|c|c|c|c|c|c|c|c|c|c|}
\hline \multirow{2}{*}{ No. } & \multirow{2}{*}{ Solvent } & \multirow{2}{*}{$\alpha^{a}$} & \multirow{2}{*}{$\pi^{* a}$} & \multicolumn{2}{|c|}{$E(F) / \mathrm{kcal} \mathrm{mol}^{-1}$} & \multirow{2}{*}{ I.P.D } & \multicolumn{2}{|c|}{$\log k_{n r}$} & \multirow{2}{*}{ I.P.D } \\
\hline & & & & Exp. & Calc. & & Exp. & Calc. & \\
\hline 1 & Methanol & 0.98 & 0.60 & 45.00 & 45.27 & 0.60 & 8.68 & 8.78 & 1.15 \\
\hline 2 & Ethanol & 0.86 & 0.54 & 45.80 & 46.25 & 0.98 & 8.88 & 8.91 & 0.34 \\
\hline 3 & 1-Propanol & 0.78 & 0.52 & 46.10 & 46.88 & 1.69 & 9.02 & 9.02 & 0.00 \\
\hline 4 & 2-Propanol & 0.76 & 0.48 & 47.70 & 47.09 & 1.28 & 9.13 & 9.07 & 0.66 \\
\hline 5 & 1-Butanol & 0.79 & 0.46 & 46.20 & 46.89 & 1.49 & 9.12 & 9.06 & 0.66 \\
\hline 6 & 1-Pentanol & 0.84 & 0.40 & 46.20 & 46.60 & 0.86 & 9.14 & 9.06 & 0.87 \\
\hline 7 & Hexanol & 0.80 & 0.40 & 47.00 & 46.90 & 0.21 & 9.15 & 9.10 & 0.55 \\
\hline 8 & Octanol & 0.77 & 0.40 & 47.60 & 47.13 & 0.99 & 9.21 & 9.12 & 0.97 \\
\hline 9 & Decanol & 0.70 & 0.45 & 47.85 & 47.58 & 0.56 & 9.25 & 9.14 & 1.19 \\
\hline 10 & Chloroform & 0.20 & 0.58 & 52.24 & 51.13 & 2.12 & 9.70 & 9.48 & 2.27 \\
\hline 11 & Acetone & 0.08 & 0.71 & 51.30 & 51.85 & 1.07 & 9.02 & 9.48 & 5.09 \\
\hline 12 & Acetonitrile & 0.15 & 0.85 & 50.40 & 51.12 & 1.43 & 9.23 & 9.31 & 0.87 \\
\hline 13 & Dichloromethane & 0.13 & 0.82 & 50.80 & 51.31 & 1.00 & 9.52 & 9.35 & 1.78 \\
\hline 14 & 2-Butanone & 0.06 & 0.67 & 53.22 & 52.05 & 2.20 & 9.69 & 9.53 & 1.65 \\
\hline \multicolumn{10}{|c|}{ Water - methanol mixture (methanol \% v/v) } \\
\hline 15 & 20.00 & 1.13 & 0.99 & 44.40 & 43.59 & 1.82 & 8.37 & 8.35 & 0.24 \\
\hline 16 & 40.00 & 1.09 & 0.89 & 44.51 & 44.03 & 1.08 & 8.46 & 8.47 & 0.12 \\
\hline 17 & 60.00 & 1.06 & 0.80 & 44.70 & 44.39 & 0.69 & 8.57 & 8.56 & 0.12 \\
\hline 18 & 80.00 & 1.02 & 0.70 & 44.82 & 44.83 & 0.02 & 8.65 & 8.67 & 0.23 \\
\hline \multicolumn{10}{|c|}{ Water - ethanol mixture (ethanol\% v/v) } \\
\hline 19 & 20.00 & 1.04 & 1.12 & 44.40 & 44.08 & 1.61 & 8.35 & 8.33 & 0.24 \\
\hline 20 & 40.00 & 0.87 & 1.03 & 44.81 & 45.48 & 1.49 & 8.59 & 8.55 & 0.46 \\
\hline 21 & 60.00 & 0.88 & 0.88 & 45.20 & 45.62 & 0.93 & 8.68 & 8.66 & 0.23 \\
\hline 22 & 80.00 & 0.89 & 0.75 & 45.41 & 45.73 & 0.70 & 8.84 & 8.75 & 1.02 \\
\hline
\end{tabular}

Table 1. Emission properties of the indicator molecule in pure and mixed solvent systems

Statistics related to the MLRA and goodness of the fit has been summarized in Table 2 (a-c). Moreover, to assess the accuracy of the equations, the experimental values of the solvatochromic properties were fitted into the equation and the mean percentage deviation (MPD) between experimental and calculated values has been estimated. For a specific data point, the individual percentage deviation (IPD) has been calculated ${ }^{26}$.

$$
\begin{gathered}
I P D=\left|\left(S P_{i}^{\exp }-S P_{i}^{c a l}\right) / S P_{i}^{\exp }\right| x 100 \\
M P D=\frac{100}{N} \sum_{i=1}^{N}\left|\left(S P_{i}^{\exp }-S P_{i}^{\text {cal }}\right) / S P_{i}^{\exp }\right|
\end{gathered}
$$

Where SP is the solvatochromic property considered $\left(E(F)\right.$ or $\left.\log k_{n r}\right)$ and $N$ is the number of the experimental data points. More than $90 \%$ of the calculated SP values have IPD less than $2 \%$. Mean percentage deviation (MPD) values have been found to be $1.13 \%$ for $E(F)$ and only $0.94 \%$ for $\log k_{n r}$. So, the accuracy of the equations has been found to be quite good. 
Table 2(a). Statistics related to the multiple linear regression analysis

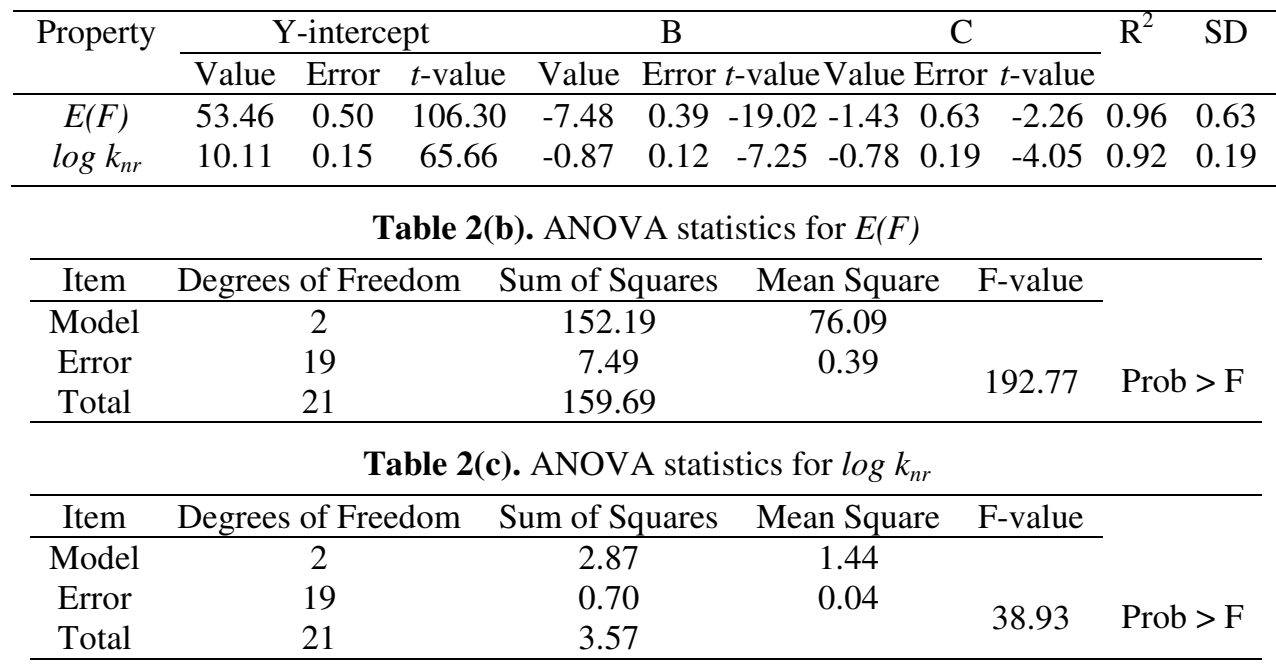

\section{In SDS micelles}

For micro-heterogeneous media like micelles, a suitable probe molecule is one which readily partitioned into micelles and whose spectral characteristics changes in some way within the micelle. The dye molecule used in the present study has been found to be an excellent probe for micellar media ${ }^{24}$. The absorption spectrum of the molecule shows two bands at $420 \mathrm{~nm}$ and $550 \mathrm{~nm}$ in SDS micellar phase. We have previously assigned the $420 \mathrm{~nm}$ band to the solubilization of the dye in some non-polar environment (near the core of the micelle) and the $550 \mathrm{~nm}$ band as for the dye molecule solubilized in some protic environment (namely the interfacial region of the micelle). It is likely that the dye molecules are distributed between the core and the interfacial region, the former is mostly hydrocarbon-like while the latter provides an environment where the hydrogen-bonded complex of the dye can exist. Steadystate fluorescence studies in SDS micellar phase show a band around 640-645 $\mathrm{nm}$ when the dye molecule has been excited to $550 \mathrm{~nm}$ light. This indicates that dye molecules are solubilised in an environment close to that of water- alkanol mixtures (octanol $600 \mathrm{~nm}$, ethanol $624 \mathrm{~nm}$, water $651 \mathrm{~nm}$ (extrapolated value)) i.e. the interfacial region of SDS micelle $^{23}$. The fluorescence band maximum shifts towards blue with increasing SDS concentration (Figure 2). This indicates gradual partitioning of the indicator into the micellar phase. But above critical micellar concentration $(\mathrm{cmc})$, the band position hardly changes i.e. constancy of emission maximum after $\mathrm{cmc}$.

In the present work, we have taken a SDS concentration of $20 \mathrm{mM}$, well above the $\mathrm{cmc}$ of SDS. The fluorescence band maximum has been found to be at $640 \mathrm{~nm}$ correspond to $E(F)=44.67 \mathrm{kcal} \mathrm{mol}^{-1}$. Moreover, when the emission is fixed at $640 \mathrm{~nm}$, the obtained excitation spectrum has been found to be identical to the absorption spectra of the dye in some protic environment. Time-resolved fluorescence study revealed that the $640 \mathrm{~nm}$ band shows double-exponential decay of the excited state with life-time $(\tau)$ components $300 \mathrm{ps}(\geq$ $25 \%)$ and $1500 \mathrm{ps}(\leq 75 \%)$ respectively. Previous work indicated that the fluorescence decay of the dye in a homogeneous media is best fitted by a single exponential term and the value of $\tau$ depends on the polarity of the solvent ${ }^{19}$. It is characterized by a longer $\tau$ in polar-protic solvents (methanol 1750 ps, ethanol 1680 ps) where the dye exists as H-bonded species. So, the observed value of 1500 ps may be assigned to the emitting state for the H-bonded species 
of the dye localized at the interfacial region of the micelle. The experimental value of $\varphi_{F}$ has been found to be 0.58 for the $640 \mathrm{~nm}$ band in $20 \mathrm{mM}$ SDS medium. Using the above two parameters $k_{n r}$ value of $2.8 \times 10^{8} \mathrm{~s}^{-1}$ has been calculated. Now one can easily obtain the $\alpha$ - and $\pi^{*}$ - values for SDS micellar media by putting the respective $E(F)$ and $\log k_{n r}$ values in the above LSER equations (1) and (2).

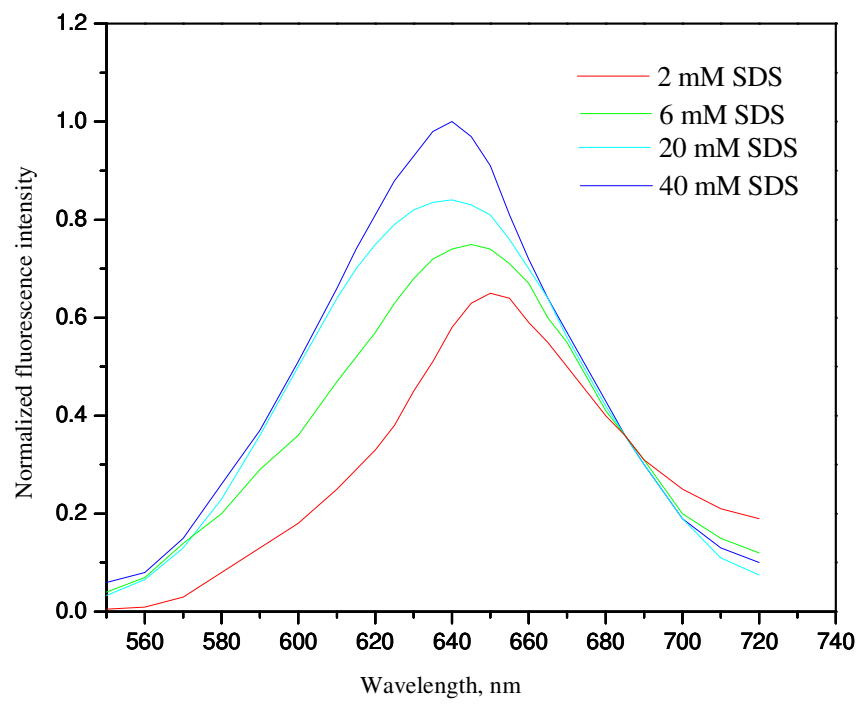

Figure 2. Fluorescence spectra of the indicator at various SDS concentrations

\section{Estimated $\alpha$ and $\pi^{*}$ of SDS micellar interface}

Using the present indicator molecule, $\alpha$-value of 0.97 and $\pi^{*}$ - value of 1.05 has been obtained for the interfacial region of SDS micelles. The obtained result needs some explanations both from qualitative and quantitative aspects. The present result of SDS system may be compared with other works on the same system also (Table 3). All the reported values are found to be smaller than that of pure water $\left(\alpha=1.17-1.19, \pi^{*}=1.09\right)$. SDS molecule do not possess any hydrogen-bond acidity on its own, the property originates from water molecules associated with the bound counter ions, headgroups and the first two methylene units of alkyl chain attached to the headgroups. So it can be inferred that associated water molecules at the interfacial region of micelles are less acidic and less dipolar than that of bulk water. The result is in accordance with the previous studies ${ }^{8,10-12}$. Solvation dynamics ${ }^{27-28}$ and Proton $\mathrm{NMR}^{29}$ studies on the structure of interfacial water of aqueous micelles has shown that they are more structured than bulk water. A water molecule at the interfacial region can take part in $\mathrm{H}$-bonding with the $-\mathrm{OSO}_{3}{ }^{-}$headgroup of the surfactant, with the neighbouring water molecules and to the basic (for our case $\mathrm{C}=\mathrm{O}$ ) part of the indicator molecule. Solvation dynamics study revealed that each headgroup is attached to water molecule through single $\mathrm{H}$-bond ${ }^{28}$. The other hydrogen atom of water may be either $\mathrm{H}$-bonded to a nearby $\mathrm{H}_{2} \mathrm{O}$ molecule or to the $\mathrm{C}=\mathrm{O}$ part of the indicator molecule. Thus the probability of $\mathrm{H}$-bond formation with the indicator molecule somewhat decreased. Thus on an average the indicator molecule senses an environment of lower acidity.

Recent molecular dynamic simulations on SDS micelle in water by Bruce et al. proposed a three shell water environment at the interfacial region ${ }^{30}$. At the third shell, outermost region from the headgroups (where radial distance between micellar headgroup 
oxygen-to-water oxygen $>6.0 \AA$ ), water molecules exhibit nearly identical behavior to that of bulk water; most of the water molecules have three hydrogen bonds followed by four and then two. At the intermediate region i.e. second shell $(3.5 \AA-6.0 \AA)$ relative ratio of four and two hydrogen bonds decreases. At the first shell $(<3.5 \AA)$ the majority of the water molecules have only two water-to-water hydrogen bonds and a significant decrease is observed in the number of water molecules with four water-to-water hydrogen bonds. Although they have found appreciable difference in water-to-water hydrogen bonding pattern of first shell water and bulk water molecules, but average number of hydrogen bonds per water molecule (including both water-to-water and micelle-to-water hydrogen bonds) differs slightly, only the weightage shifted towards fewer number of hydrogen bonds. So, it is expected that first shell water molecules and bulk water should not differ by too large extent in their hydrogen bond donating abilities. Thus $\alpha$-value of 0.97 is reasonable from that view point.

The $\pi^{*}$-value of 1.05 indicates that the indicator molecule is exposed to an environment of large water content, not to any non-polar region. The value supports the fact that the indicator molecule resides at the first shell of the interfacial region.

\section{Effect of methodology and choice of indicator molecules}

Table 3 summarizes all the reported values of $\alpha$ and $\pi^{*}$ for SDS micellar media. $\alpha$ - values range from 0.8 to 1.1 whereas a larger spread $(0.5-1.1)$ has been observed for $\pi^{*}$ - values. Regarding the methodology used in the estimation process it is found that apart from Vitha et al., ${ }^{31}$ that applied Kamlet-Taft solvatochromic comparison method based on the behavior of pairs of indicators, all the other workers have used the multiparametric equation as developed by Marcus ${ }^{32,33}$. So, the observed spread can be assigned partly to the difference in methodology used. But even the estimated values from similar methodologies differ. Thus it is evident that choice of indicator molecule plays a role here.

Table 3. Reported $\alpha$ and $\pi^{*}$ - values of SDS micellar phase

\begin{tabular}{|c|c|c|c|}
\hline Ref. & $\begin{array}{l}\text { Solvatochromic } \\
\quad \alpha\end{array}$ & $\underset{\pi^{*}}{\text { Properties }}$ & Indicator molecule (s) used \\
\hline $\begin{array}{l}\text { Deb et al. } \\
\text { (Ref. 13) }\end{array}$ & 1.18 & 0.52 & $\begin{array}{l}\text { A ketocyanine dye and pyrene } \\
\text { (fluorescence probes) }\end{array}$ \\
\hline $\begin{array}{c}\text { Shannigrahi } \\
\text { et al. } \\
\text { (Ref. 11) }\end{array}$ & 1.14 & 0.52 & $\begin{array}{l}\text { A series of structurally similar } \\
\text { ketocyanine dyes (fluorescence probes) }\end{array}$ \\
\hline $\begin{array}{l}\text { Fuguet et al. } \\
\text { (Ref. 10) }\end{array}$ & 0.79 & $1.02(\mathrm{av})$. & $\begin{array}{c}\text { ET(33) dye and a series of } \pi^{*} \text {-indicators } \\
\text { (absorption probes) }\end{array}$ \\
\hline $\begin{array}{l}\text { Vitha et al. } \\
\text { (Ref. 8) }\end{array}$ & $\begin{array}{l}0.73,1.01 \\
0.87 \text { (av.) }\end{array}$ & $1.06(\mathrm{av})$. & $\begin{array}{c}\mathrm{ET}(33) \text { dye, } \mathrm{Fe}(\mathrm{LL})_{2}(\mathrm{CN})_{2} \text { and a series } \\
\text { of } \pi^{*} \text {-indicators (absorption probes) }\end{array}$ \\
\hline $\begin{array}{l}\text { De Costa et al. } \\
\quad \text { (Ref. 34) }\end{array}$ & 0.82 & 0.52 & $\begin{array}{l}\text { 1,5-diphenyl-3-vinyl- } \Delta^{2} \text {-pyrazoline } \\
\text { (fluorescence probe) }\end{array}$ \\
\hline
\end{tabular}

Although the present result corroborates well to that of Vitha et al. ${ }^{8}$ and Fuguet et al. ${ }^{10}$ but it is better to compare the present work to that of De Costa et $a l^{34}$. for their similar methodology and as both have used single indicator molecule. They have used two environment sensitive emission properties (maximum energy of fluorescence and quantum yield) of a single indicator molecule 1,5-diphenyl-3-vinyl- $\Delta^{2}$-pyrazoline for that purposes. They have estimated $\alpha$-value of 0.82 and $\pi^{*}$ - value of 0.52 . The $\alpha$-value has been found to 
be quite close to that of the present work, but $\pi^{*}$ - value differ by two folds. It should be noted that the empirical relations used by them were based on fewer data points $(n=4$ in case of quantum yield and $n=10$ in case of maximum energy of fluorescence) compared to the present study $(n=22)$. But even then, the observed spread in the estimated properties may be assigned to the following two facts. Firstly, due to the differences in the locus of solubilization of the indicator molecule (s) within the micelle. Secondly, differences in the chemistry of the indicator (s) used. This is probably originates from the relative weightage of the dye towards the basic modes of solvation i.e. on the ratio $\left(\alpha / \pi^{*}\right.$ value of the LSER equation) for a specific indicator.

\section{Conclusion}

The present work summarizes a new methodology for the estimation of solvatochromic properties of micellar interface. Two extremely environment sensitive parameters, viz., energy of fluorescence maximum, $E(F)$ and the non-radiative decay constant of the excited state, $k_{n r}$ of a single ketocyanine dye has been used for that purpose. The hydrogen bond donating ability and dipolaity-polarisability of associated water molecules at the SDS micellar interface has been found to be 0.97 and 1.05 respectively. As expected the values are to some extent lower than that of bulk water. Moreover, both the properties have some dependency on the choice of indicator molecule.

\section{Acknowledgement}

The author thanks Department of Chemistry, The University of Burdwan, India for providing infrastructural facilities. The author is also thankful to Prof. S. Bagchi for his inspiration and guidance.

\section{References}

1. Fendler J H and Fendler E J, Catalysis in Micellar and Macromolecular Systems, Academic Press, New York, 1975.

2. Rosen M J, Surfactants and Interfacial Phenomena, John Wiley and Sons, New York, 1978.

3. Pramauro E and Pelizetti E, Surfactants in Analytical Chemistry; Applications of Organized Amphiphilic Media, Elsevier, Amsterdam, 1996.

4. Terabe S, Otsuka K and Ando T, Anal Chem., 1985, 57, 834-841.

5. $\quad$ Rideout D C and Breslow R, J Am Chem Soc., 1980, 102, 7816-7817.

6. $\quad$ Engberts J B F N, Pure Appl Chem., 1992, 94, 653.

7. Kamlet M J, Abboud J L M, Abraham M H and Taft R W, J Org Chem., 1983, 48, 2877-2887.

8. Vitha M F, Weckwerth J D, Odland K, Dema V and Carr P W, J Phys Chem., 1996, 100, 18823.

9. Vitha M F and Carr P W, J Phys Chem B, 1998, 102, 1888.

10. Fuguet E, Rafols C, Bosch E and Roses M, Langmuir, 2003, 19, 55-62.

11. Shannigrahi M and Bagchi S, J Phys Chem B, 2004, 108, 17703-17708.

12. Shannigrahi M and Bagchi S, J Phys Chem B, 2005, 109(30), 14567-14572.

13. Deb N, Shannigrahi M and Bagchi S, J Phys Chem B, 2008, 112(10), 2868-2873.

14. Laurence C, Nicolet P, Dalati M T, Abboud J L M and Notario R, J Phys Chem., 1994, 98, 5807-5816.

15. Kessler M A and Wolfbeis O S, Spectrochim Acta Part A, 1991, 47(2), 187-192.

16. Tatikolov, Krasnaya A Zh, Ahvedova L A and Kuzmin V A, Int J Photoene., 2000, 2(1), 23-30. 
17. Baraldi I, Brancolini G, Momicchioli F, Ponterini G and Vanossi D, Chem Phys., 2003, 288, 309-325.

18. Banerjee D, Laha A K and Bagchi S, Indian J Chem., 1995, 34A, 94.

19. Banerjee D and Bagchi S, J Photochem Photobiol A, 1996, 101, 57-62.

20. Banerjee D, Mondal S, Ghosh S and Bagchi S, J Photochem Photobiol A, 1995, 90(2-3), 171-176.

21. Pramanik R, Das P K and Bagchi S, Phys Chem Chem Phys., 2000, 2, 4307-4311.

22. Ray N and Bagchi S, J Mol Liq., 2004, 111(1-3), 19-24.

23. Banerjee D, Das P K, Mondal S, Ghosh S and Bagchi S, J Photochem Photobiol A, 1996, 98(3), 183-186.

24. Shannigrahi M and Bagchi S, J Photochem Photobiol A, 2004, 168(3), 133-141.

25. Shannigrahi M and Bagchi S, Chem Phys Lett., 2005, 403(1-3), 55-61.

26. Habibi-Yangjeh A, Bull Korean Chem Soc., 2004, 25(8), 1165-1170.

27. Bhattacharyya K and Bagchi B, J Phys Chem B, 2000, 104, 10603.

28. Balasubramanian S, Pal S and Bagchi B, Curr Sci., 2002, 82(7), 845-854.

29. El Seoud O A, Bazito R C and Barlow G K, Progr Colloid Polym Sci., 1998, 111, 151-157.

30. Bruce C D, Senapati S, Berkowitz M L, Perera L and Forbes M D E, J Phys Chem B, 2002, 106(42), 10902-10907.

31. Kamlet M J and Taft R W, J Am Chem Soc., 1976, 98, 377-383.

32. Marcus Y, Chem Soc Rev., 1993, 22, 409-416.

33. Marcus Y, J Chem Soc Perkin Trans 2, 1994, 1751.

34. De costa M D P, De Silva A P and Pathirana S T, Can J Chem., 1987, 65, 1416-1419. 